\title{
How to Measure the Content of Hazardous Substances in Food with the High Performance Liquid Chromatography-Mass Spectrometry Combination Method
}

\author{
Ying An \\ School of Chemical Engineering, Xuzhou College of Industrial Technology, Xuzhou Jiangsu, 221140, \\ China
}

Keywords: HPLC-MS, Food, Hazardous substances, Ethoxyquin antioxidant

\begin{abstract}
Purpose: Analyze the results of measuring the content of hazardous substances in food with the high performance liquid chromatography (HPLC)-mass spectrometry (MS) combination method. Method: (1) Establish the HPLC-MS to achieve the one-step extraction of the ethoxyquin antioxidant residue in honey peach and apple by extracting the analyte residue in alkaline sample solution with the help of N-hexane; then analyze it with the C18 chromatographic column and add a certain amount of ammonium acetate into the mobile phase to optimize the peak shape of chromatogram. (2) Establish the HPLC-MS to detect urotropin in food. Results: (1) It is found that the lower limit of detection with the HPLC-MS combination method is $5 \mu \mathrm{g} / \mathrm{kg}$, and the linearity range is between 20-200 $\mu \mathrm{g} / \mathrm{L}$; all the recovery rates of different adding levels are between $8.65 \%-91.9 \%$, and the RSD (relative standard deviation) is between 4.6\%-5.4\%. (2) For the HPLC-MS combination method, the $0.01 \mathrm{~mol} / \mathrm{L}$ ammonium acetate-acetonitrile is the mobile phase, which provides high accuracy and sensitivity, fits the treatment of a lot of samples, and is used for detecting urotropin to protect the health of consumers. Conclusion: The HPLC-MS combination method is highly feasible in measuring the content of hazardous substances in food.
\end{abstract}

\section{Introduction}

Currently, there are many methods to detect hazardous substances in food, and common ones include gas chromatography combined with nitrogen-phosphorus detector (NPD) or HPLC coordinated with ultraviolet ray. With the development of detection technology, the HPLC-MS combined technique gets its promotion, has high sensitivity and ability of nature determination, and is widely applied in detecting and analyzing various pesticides and residues in food.

\section{Test of detecting ethoxyquin in fruits}

Currently, ethoxyquin is one of the most widely used efficient antioxidants in the world, has a strong function of freshness preservation and antisepsis, and is often used for freshness preservation of fruits such as apple and peach ${ }^{[1]}$. According to hygienic standards related to food additives in China, ethoxyquin can be used as preservative for surface-treated fruits, but its amount of usage should be strictly controlled and the amount of residue should be within $1 \mathrm{mg} / \mathrm{kg}$. The amount of residue recommended by the Codex Alimentarius is $3 \mathrm{mg} / \mathrm{kg}$, while it is stipulated to be $0.05 \mathrm{mg} / \mathrm{kg}$ by EU.

\section{Instruments and reagents}

Select Germany-made liquid chromatogram (LC)-high resolution tandem quadrupole mass spectrometer, analytically pure sodium hydroxide, redistilled water, chromatographically pure methanol, chromatographically pure ammonium acetate, ethoxyquin whose purity exceeds $98 \%$, etc.

\section{Standard solution preparation}

Use the methanol and ethoxyquin sample solution to prepare $1.0 \mathrm{~g} / \mathrm{L}$ monostandard stock solution and have it cryopreserved in dark area, in accordance with the operating instructions. Adopt the actual starting gradient of mobile phase for proportioning, as needed by the actual detection, till the stock solution is diluted to the range of $1.2-200.0 \mu \mathrm{g} / \mathrm{L}$. 


\section{Detection conditions}

The first one is the chromatographic condition: the chromatographic column is $50 \mathrm{~mm} \times 2.1 \mathrm{~mm} \times$ $1.9 \mu \mathrm{m}$; there are two kinds of mobile phases: mobile phase $\mathrm{A}$ is the ammonium acetate aqueous solution with the concentration of $5 \mathrm{mmol} / \mathrm{L}$ and the formic acid aqueous solution with the concentration of $0.1 \%$; mobile phase $\mathrm{B}$ is the methanol; the column temperature is the room temperature; the sample injection volume is set to be $25 \mu \mathrm{L}$.

The second one is the MS condition: adopt the positive-ion detection method, for which the voltage is $10 \mathrm{~V}$; the parent ion condition of ethoxyquin is $218.1 \mathrm{~m} / \mathrm{z}$, and its daughter iron condition is $147.9 / 175.9$; the cone voltage is $50 \mathrm{~V}$.

\section{Sample weighing}

Accurately weigh $5.00 \mathrm{~g}$ sample and place it into a conical flask with a plug, and then add $15 \mathrm{ml}$ sodium hydroxide solution with the concentration of $0.1 \mathrm{~mol} / \mathrm{L}$ as well as $50 \mathrm{ml} \mathrm{N}$-hexane; mix them by shaking the conical flask for 20 minutes; transfer the prepared extraction solvent into a flask; repeat the above steps and merge the N-hexane extraction solvent; then, have it dried through rotation in the $35^{\circ} \mathrm{C}$ water bath; Mobile phase ammonium acetate solution: the proportion of Formic acid aqueous solution and methanol is $35: 65$, and the constant volume is $1.0 \mathrm{ml}$; filtrate it with the $0.45 \mu$ $\mathrm{m}$ filter membrane and then transfer it into the sample injection bottle for detection.

\section{Content of ethoxyquin in fruits}

\section{Selecting suitable extraction solvents}

There is quinoline ring in the chemical formula of ethoxyquin, which is a kind of weak polar compound and is soluble in organic solvents but not soluble in water; therefore, such organic solvents as methanol, N-hexane and acetonitrile are used for intervention in this test. The results showed that using any one of the solvents for direct extraction is low-efficiency; the efficiency of extraction using methanol reaches just $10 \%$, and highest efficiency of extraction using $\mathrm{N}$-hexane is just $42 \%$. As this test involves fruits such as apple and honey peach that contain a large amount of moisture, using methanol and the like as extraction solvents will lead to difficulty in concentration, and the recovery rate is still low even after adding dehydrating reagents such as magnesium sulfate. The main cause leading to this phenomenon is that quinoline ring can receive protons; as a kind of weak organic base, ethoxyquin presents in the ionic form in fruit mechanism, resulting in low-efficiency extraction of organic solvents ${ }^{[2]}$. In some research maps of the above test, the recovery rate of adding $5 \mathrm{ml}$ sodium carbonate solution and then using $\mathrm{N}$-hexane for extraction reaches $90 \%$; its mechanism lies in the weak alkaline solution that can restrain the ionization of relevant substances in ethoxyquin, thus the recovery rate is improved. This practice is kept in this research; meanwhile, the HPLC-MS combined their own characteristics, providing strong anti-jamming capability and simplifying the pretreatment process efficiently; in this group, sodium hydroxide solution is used to restrain ionization, which finally leads to high recovery rate.

In this group, the actual fortified concentration is $100 \mu \mathrm{g} / \mathrm{kg}$, and sodium hydroxide solutions of 4 kinds of concentrations $(0.05 \mathrm{~mol} / \mathrm{L}, 0.10 \mathrm{~mol} / \mathrm{L}, 0.20 \mathrm{~mol} / \mathrm{L}$ and $0.50 \mathrm{~mol} / \mathrm{L})$ are added to compare the difference in recovery rates of using $\mathrm{N}$-hexane for extraction. The final results showed that the sodium hydroxide solutions of 4 different concentrations have little influence on extraction using $\mathrm{N}$-hexane; the extraction efficiency of the $0.10 \mathrm{~mol} / \mathrm{L}$ sodium hydroxide solution is about $86 \%$, and that of the other three concentrations of sodium hydroxide solutions is just between $78 \%$ and $82 \%$ respectively. Thus, the $0.10 \mathrm{~mol} / \mathrm{L}$ is finally selected as the test concentration.

\section{Influence of mobile phase}

The test showed that using methanol as the mobile phase can improve the sensitivity in a better way. Compared with acetonitrile, methanol increased the sensitivity by $20 \%$ when it is used as the organic phase; meanwhile, adding a certain amount of acid or volatile dielectric in options will only affect the retention time and peak shape of the analyte. In this group of tests, adding $0.1 \%$ formic acid can bring forward the chromatographic peak and compress its width, but the leading peak may also appear; while adding acetic acid can only keep the symmetry of peak shape and its width is big, thus the sensitivity is also lower ${ }^{[3]}$. When both formic acid and acetic acid are added during the test, it is found that there is no leading peak but good symmetry and that there is no obvious difference in 
sensitivity when compared with the use of formic acid only; methanol is finally selected as the mobile phase, while a suitable amount of formic acid and ammonium acetate are added in aqueous phase.

Besides, different reversed phase columns are used in the research, and the results showed that the weak acute characteristic of ethoxyquin results in very strong retention in common C18 chromatographic column; a good peak shape can be guaranteed to improve the test efficiency only by using very efficient organic phase ratios and also strengthening the control of $\mathrm{PH}$ value of mobile phase. For this case, short columns that are rarely used in common tests are adopted in this group of tests, so that analyte on chromatographic column can be eluted in a very shot time, saving a lot of time and improving the test efficiency substantially.

Linearity range, recovery rate, accuracy, etc. of detection

Use mobile phase for preparation to obtain standard solution whose mass concentration is between 1.0-200 $\mu \mathrm{g} / \mathrm{L}$; determine the correlation coefficient of ethoxyquin linear equation to be 0.993 ; add the prepared ethoxyquin standard solutions ( $5 \mu \mathrm{g} / \mathrm{kg}, 10 \mu \mathrm{g} / \mathrm{kg}$ and $20 \mu \mathrm{g} / \mathrm{kg}$ respectively) in matrixes of honey peach and apple; repeat the measurement for 10 times to obtain the detection accuracy and recovery rate. When the adding level is $5 \mu \mathrm{g} / \mathrm{kg}$ : the average measured value, average recovery rate and RSD of honey peach are $4.35 \mu \mathrm{g} / \mathrm{kg}, 86.3 \%$ and 5.2 respectively, while those of apple are 4.43 $\mu \mathrm{g} / \mathrm{kg}, 88.5 \%$ and 5.0 respectively; when the adding level is $10 \mu \mathrm{g} / \mathrm{kg}$ : those of honey peach are $4.72 \mu \mathrm{g} / \mathrm{kg}, 86.9 \%$ and 5.0 respectively, while those of apple are $8.91 \mu \mathrm{g} / \mathrm{kg}, 89.6 \%$ and 5.1 respectively; when the adding level is $20 \mu \mathrm{g} / \mathrm{kg}$ : those of honey peach are $17.48 \mathrm{\mu} \mathrm{g} / \mathrm{kg}, 88.7 \%$ and 4.8 respectively, while those of apple are $18.21 \mu \mathrm{g} / \mathrm{kg}, 91.2 \%$ and 4.7 respectively.

\section{Test of detecting urotropin in food}

\section{Instruments and reagents}

Apply the Germany-made fast liquid chromatograph for detection; select the urotropin whose purity is over $99 \%$, chromatographically pure methanol and acetonitrile, ammonium acetate whose purity is over $98 \%$, acetic acid whose purity is over $99.5 \%$, formic acid whose purity is over $98 \%$, analytically pure pearl barley, and analytically pure ethyl alcohol.

\section{Standard solution preparation}

Accurately weigh urotropin and transfer it into the $100 \mathrm{ml}$ volumetric flask; add acetonitrile for dissolving and diluting into $100 \mathrm{mg} / \mathrm{L}$ standard solution. Precisely absorb $1 \mathrm{ml}$ solution into the $100 \mathrm{ml}$ volumetric flask for further dilution with acetonitrile into $1 \mathrm{mg} / \mathrm{L}$ midst standard solution, and then dilute it into 1.0-500 $\mu \mathrm{g} / \mathrm{L}$ standard solution; filtrate it with the $0.22 \mu \mathrm{m}$ filter membrane and get it ready for detection.

\section{Sample pretreatment}

Weigh $3 \mathrm{~g}$ sample and transfer it into a centrifugal tube, and then add $2.0 \mathrm{~g}$ anhydrous sodium sulfate and $10 \mathrm{ml}$ acetonitrile for vortex mixing; then conduct the high-speed centrifugation for 5 minutes, and filtrate the supernatant liquid for use.

\section{Results}

Among mass spectrum conditions, parameters such as capillary voltage, dryer temperature and flow rate are determined based on relevant reference values of instruments. In the positive ion mode, there are small conditions; scan the parent ions of target compound of urotropin solution with the concentration of $1 \mathrm{mg} / \mathrm{L}$, optimize the parameter of outlet voltage and then optimize the collision energy, so as to determine $\mathrm{m} / \mathrm{z} 42$ as quantitative ion.

Acetonitrile-ammonium acetate (PH3.5), acetonitrile-ammonium acetate (PH5.0) and acetonitrile-ammonium acetate (PH6.8) are used as mobile phases in the test. When acetonitrile-ammonium acetate (PH3.5) is used: the sample has a symmetrical peak shape and a long retention time, which is about 10 minutes; when acetonitrile-ammonium acetate (PH6.8) is used: the retention time of sample is 1.5 minutes, and the tailing phenomenon exists. Therefore, the acetonitrile-ammonium acetate (PH3.5) is selected as the mobile phase. The gradient elution is adopted for observation, and it is finally determined that good response value and ion strength are 
obtained when the initial proportion of mobile phase, namely the $0.01 \mathrm{~mol} / \mathrm{L}$ ammonium acetate, is $70 \%$; thus, it is taken as the initial proportion of mobile phase.

Finally, the detection limit of 3 times $\mathrm{S} / \mathrm{N}$ determination method is $0.2 \mu \mathrm{g} / \mathrm{kg}$, and that of 10 times $\mathrm{S} / \mathrm{N}$ determination method is $1.0 \mu \mathrm{g} / \mathrm{kg}$; select the standard solution with the concentration of $100 \mu$ $\mathrm{g} / \mathrm{L}$ and random sample for 6 repeated detections; RSD is between 1.1\% and 3.6\%, and the instrument precision is good; besides, the adding standard recovery rate is between $53.1 \%$ and $116.8 \%$, and RSD is below $10.1 \%$. As to the wet rice noodle, the actual measured value, recovery rate and RSD are $0.868 \mu \mathrm{g} / \mathrm{kg}, 73.2 \%$ and 9.2 respectively when $1.2 \mu \mathrm{g} / \mathrm{kg}$ urotropin is added; those are $2.783 \mu \mathrm{g} / \mathrm{kg}$, $56.1 \%$ and 7.4 respectively when $5.0 \mu \mathrm{g} / \mathrm{kg}$ urotropin is added; those are $26.83 \mu \mathrm{g} / \mathrm{kg}, 53.3 \%$ and 8.5 respectively when $50.0 \mu \mathrm{g} / \mathrm{kg}$ urotropin is added.

\section{Discussion}

Food is one of the indispensable things in human life, and different foods have different nutritional ingredients and characteristics; their ingestion concerns people's health ${ }^{[4]}$. With the development of modern work and the purpose of improving food quality, it is required to add relevant chemical agents to adjust their elements such as color and flavor or to satisfy the needs of corrosion prevention and antibiosis; excessive adding of such chemical agents may harm human health, thus adding of such agents should be within a limited range. Besides, food may be also polluted during planting, production, processing and transportation due to human-related or non-human factors, such as pesticide residue, adding of industry dyes, etc., which threatens human health ${ }^{[5]}$. Therefore, detecting hazardous substances in food by appropriate means is very critical for promoting the development of food safety. In this group of tests, the HPLC-MS combined technique is adopted to detect ethoxyquin in honey peach and apple as well as urotropin in wet rice noodle, both of which showed good accuracy and sensitivity. In a word, the HPLC-MS combined technique integrates the HPLC's ability of separating complex matrixes and MS's high sensitivity and selectivity, is widely used in food quality safety analysis and provides an effective means of market monitoring.

\section{Acknowledgments}

Xuzhou Technology Project, which name is Detection and Shifting Law Research on Toxic Substance in Food Contact Materials, Project No. KC14SM085.

\section{References}

[1] Zhu Yamei, Zhu Chengjing, Li Wen, etc. Measurement of Carbendazim Residues in Fruits and Vegetables Using HPLC-Fluorescence Detection Method. Memoir of the Fifth Chromatography and Mass spectrometry Symposium in Eastern China. 2012: 1-4.

[2] Jiang Yuan, Shen Chongyu, Yao Yigang, etc. Measurement of Sulfonamides Potentiator Residue in Animal Derived Food Using HPLC-Tandem MS Method. Journal of Instrumental Analysis, 2009, 28(7): 834-837, 841.

[3] Ding Tao, Shen Chongyu, Jiang Yuan, etc. Measurement of Ethoxyquin Residue in Fruits Using HPLC-Tandem MS Method. Journal of Chinese Mass Spectrometry Society, 2009,30(5):307-310.

[4] Zhu Chengjing, Wang Xiaojuan, Zhao Houmin, etc. Research on Measurement of Carbendazim Residue in Leaf Vegetables Using HPLC Method. Memoir of the Fourth Chromatography and Mass spectrometry Symposium in Eastern China. 2010: 139-143.

[5] Zhang Housen, Wu Xiaojing, Wang Wei, etc. Uncertainty Evaluation of Sulfadoxine in Pork Using HPLC Tandem MS method. Food Engineering, 2012,(3): 55-58. 